\title{
POLARISATION-SENSITIVE OPTICAL COHERENCE TOMOGRAPHY FOR MATERIAL CHARACTERISATION AND TESTING
}

D.Stifter ${ }^{1}$, A.D.Sanchis Dufau ${ }^{1}$, E. Breuer ${ }^{1}$, K. Wiesauer ${ }^{1}$, P.Burgholzer $^{1}$, O.Höglinger ${ }^{1}$, E.Götzinger ${ }^{2}$, M.Pircher ${ }^{2}$, C.K.Hitzenberger ${ }^{2}$

${ }^{1}$ Upper Austrian Research GmbH, Linz, Austria; ${ }^{2}$ Department of Medical Physics, Medical University of Vienna, Vienna, Austria

\begin{abstract}
Optical Coherence Tomography (OCT) is an emerging technology for high-resolution non-contact imaging of semitransparent structures. Originally developed for medical diagnostics, we extend the OCT-technique to problems posed in material testing and characterization. Layer thickness and refractive indices as well as internal structures of polymer parts have been determined within this study. An extension of OCT, namely polarisation-sensitive OCT (PSOCT) has been used to identify regions of increased anisotropy within injection-moulded plastic parts and to determine quantitatively internal strain within translucent materials in a nondestructive way.

Introduction and experimental: Optical Coherence Tomography (OCT) is an emerging technology for high-resolution non-contact imaging of semitransparent structures. The measurement principle of OCT is based on an interferometric detection of path-length distributions of low-coherence light back-reflected from interfaces within the sample [1-3]. The original application of OCT is the imaging of the human retina [1] and has been extended to the characterisation of a variety of biological tissues [3]. Anyhow, the applications of OCT outside the biomedical sector, especially for material characterisation and testing are so far only marginally touched: e.g. OCT imaging of glass fibre composites and the detection of subsurface defects and cracks in other non-biological materials, like in ceramics [4,5].

Polarisation-Sensitive OCT (PS-OCT) is an extension to the classical OCT method and maps - in addition to the light intensity distribution - the polarisation state of light within the sample [6-8]. Thus, additional physical parameters (like birefringence) and enhanced structural information, that is difficult to resolve with other imaging techniques, can be obtained.

Recently, we extended for the first time classical OCT measurements to PS-OCT to characterise and non-destructively test injection-moulded polymer parts for a qualitative determination of internal strain fields and regions of material anisotropy [9]. In the current study we present new applications of classical OCT, like non-destructive thickness measurements of lacquer-layers and discrimination between individual layers, even when of same consistence and composition. Furthermore, we performed, simultaneously to PS-OCT, tensile stress-strain experiments on different polymeric materials to measure the stress-optical coefficient in the perspective of a quantitative and non-destructive determination of residual and induced internal stress in samples. For the presented study we used a PS-OCT set-up as schematically depicted in Fig. 1. The beam of a low coherent light source is linearly polarised (vertically) and divided equally into two arms of a Michelson interferometer. In the reference arm a scanning mirror varies the optical path length. The quarter wave plate in the reference arm provides after back-reflection from the mirror linearly polarized light rotated by $45^{\circ}$ to its original polarisation direction. The sample under investigation is placed in the other arm and is illuminated by circularly polarized light with the help of a second quarter wave plate.
\end{abstract}




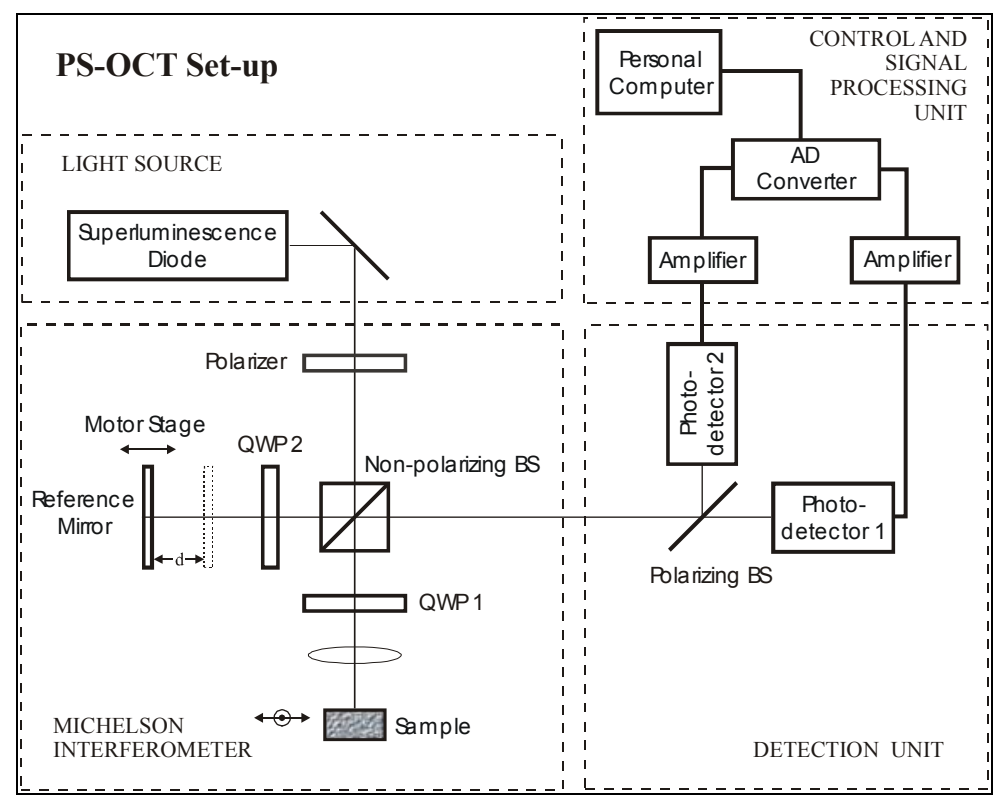

Fig. 1: Schema of PS-OCT set-up with broadband light source, Michelson interferometer, detection and processing unit (BS: beam splitter; QWP: quarter wave plate).

Optical inhomogeneities (e.g. refractive index changes) within the sample reflect and scatter back parts of the incoming light, which is combined with the reference beam at the non-polarising beam splitter of the interferometer. Interference can only occur, if the optical path length of the reference arm equals the path length from the interferometer beam splitter to a reflective interface within the sample. The interference signal (at a Doppler frequency determined by the scanning speed of the reference mirror) is recorded as a function of depth, separately for the two orthogonal polarisation directions (vertical and horizontal) using a polarising beam splitter and two detector units. Therefore, one depth scan (or so-called optical A-scan), carried out by the movement of the reference mirror, provides a one-dimensional distribution of sample reflectivity for both polarisation directions along the z-axis. From the intensity and relative phase of the two interference oscillations the reflectivity, the birefringence and the orientation of the fast optical axis can be simultaneously acquired in a spatially resolved way [8]. The axial (depth) resolution of this method is determined by the coherence length of the used light source [10] and lies typically around 10-20 $\mu \mathrm{m}$ for standard superluminescence diodes (SLD) and amplified spontaneous emission (ASE) sources. By performing A-scans at laterally adjacent sample positions, 2D and 3D information is gained. Therefore, the lateral resolution is limited by the spot size of the focused beam on the sample.

OCT and PS-OCT measurements have been performed by us with a specially for material investigation designed set-up: a $3 \mathrm{~mW}$ SLD emitting at a centre-wavelength of $1550 \mathrm{~nm}$ has been employed in order to increase the penetration depth in higher scattering materials. The SLD exhibited a spectral width (full width half maximum, FWHM) of about $55 \mathrm{~nm}$ resulting in a round trip coherence length of $\sim 19 \mu \mathrm{m}$ in air and $\sim 14 \mu \mathrm{m}$ in polymer materials, assuming a mean refractive index around 1.4 .

Results and Discussion: OCT - due to its characteristics to measure in reflection - is highly suitable for thickness evaluations of coatings, films and layers. As a first example serves fig. 2 in which the cross-sectional OCT image of a painted wooden surface is presented. The wooden surface has been coated with one layer of transparent synthetic resin paint, dried for 12 hours and repainted. Although both layers are nominally of same consistence and composition, they can be 
clearly discerned, important for quality control of subsequently painted and repainted surfaces. Optical inhomogeneities at the interface between the two layers cause a detectable OCT-signal, which may originate from small dust particles, air inclusions or variation of composition at the dried and repainted surface. Our setup provides at the moment a depth resolution of around $14 \mu \mathrm{m}$, as mentioned in the above section. The application of a light source with a broader spectrum will clearly enhance the precision in the determination of the individual interfaces. In addition, the interface between resin paint and wooden surface needs closer and further examination: in fig. 2, varying depths of the penetration of the resin paint into the wooden material can be detected.

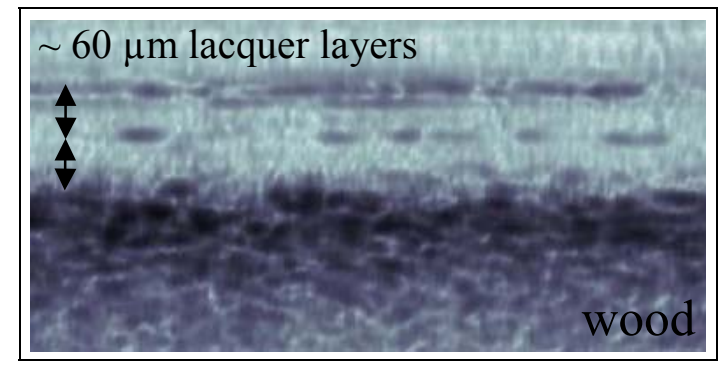

Fig. 2: OCT cross-sectional image of 2 layers of synthetic resin paint on wooden surface (each approx. $60 \mu \mathrm{m}$ ).

When evaluating OCT images, the measurement principle has to be taken into account. Since OCT does not directly measure geometrical distances but optical path lengths, caution has to be applied for non-flat surfaces and interfaces. In fig. 3 (left side) a semitransparent layer of polyurethane elastomer covering half the area of a flat fibre composite substrate is depicted.

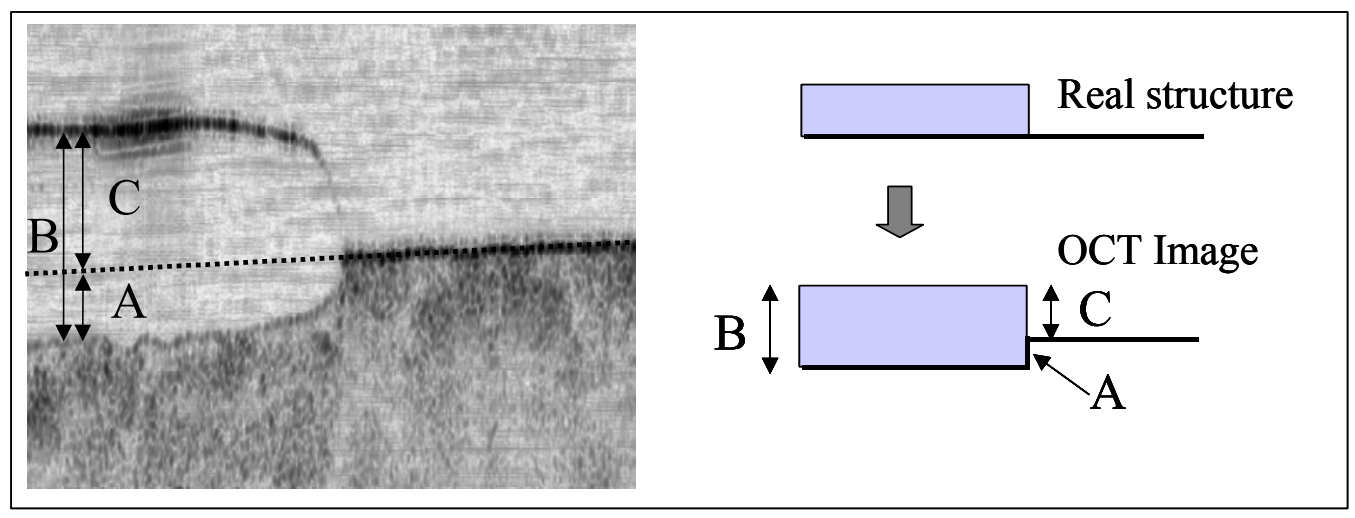

Fig.3: OCT image of thin film (polyurethane elastomer) covering partially a plane substrate surface (fibre composite). The path experienced by the investigating light beam creates a virtual step in the substrate surface (schema depicted on right side with A, B, C marking measurable differences of optical pathlengths)).

The light which has to travel trough the layer experiences an optical path which equals the layer thickness times the refractive index of the layer. For the uncovered surface region the light has to travel the same geometrical distance in air with refractive index $=1$. This fact causes a virtual step in the substrate surface as schematically depicted on the right side of fig. 3 . The disadvantage of a "distorted" picture may be compensated by the determination of an additional sample parameter. By applying simple geometrical considerations, one can easily determine true geometrical layer thickness and refractive index (at center wavelength of light source) 
simultaneously. In the above example, the determination of optical path lengths $(B=555 \mu \mathrm{m}, \mathrm{C}=$ $365 \mu \mathrm{m} ; \mathrm{A}=\mathrm{B}-\mathrm{C})$ yields a geometrical layer thickness of $365 \mu \mathrm{m}$ and a refractive index $\mathrm{n}=1.52$. The accuracy is again depending on the width and shape of the light spectrum and the flatness of the substrate surface.

The same principle can be applied not only for layers but also bulk materials. In fig. 4 the OCTintensity profile measured at two different locations of a bulk polymer sample is depicted (solid line and dotted line). The sample has a corrugated top surface and a flat backsurface resulting in a virtually corrugated back surface.

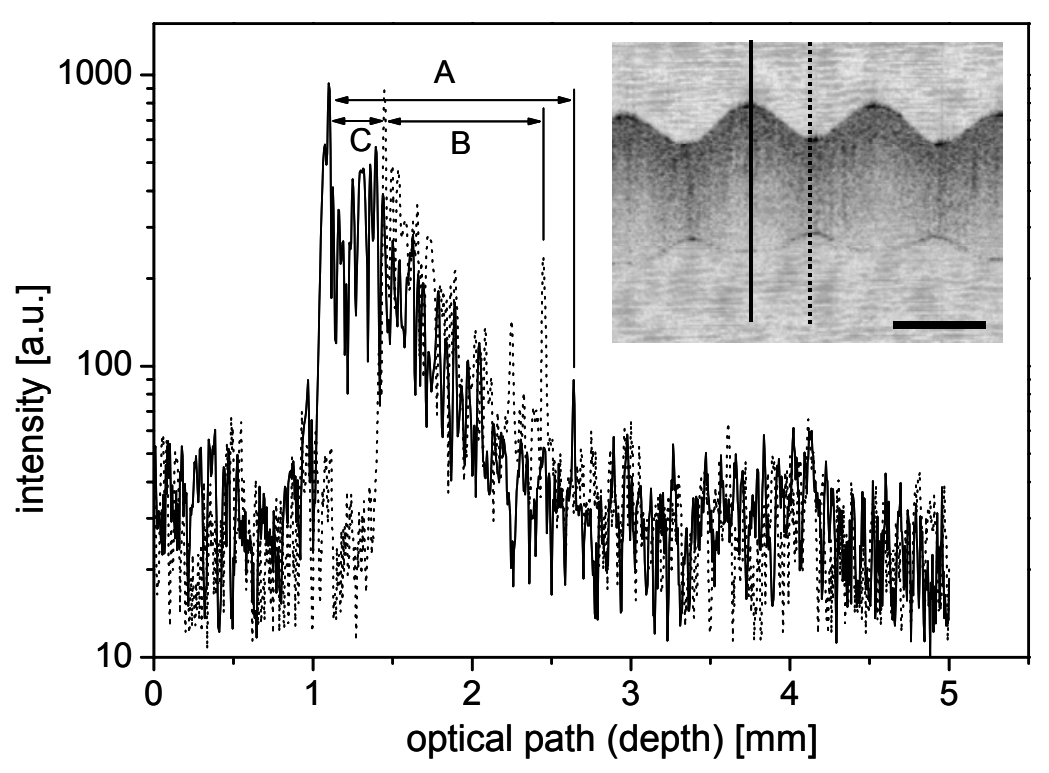

Fig.4: OCT cross-sectional image of a polymer sample with corrugated top-surface and flat back surface. Measurement of optical path lengths A, B, C enable determination of geometrical thickness and refractive index. The two plotted lines give the intensity profile at two different sample positions, as indicated in the inset (full line, dotted line). Horizontal scale bar: $1 \mathrm{~mm}$

By detecting the exact peak positions of the back and front surfaces and taking $n=(A-B) / C$, a refractive index of $\mathrm{n}=1.54$ and geometrical distances of $1 \mathrm{~mm}$ (thickness of sample at solid line) and $0.54 \mathrm{~mm}$ (thickness at dotted line) can be determined. With our setup, we can provide an edge (peak) detection accuracy around $5 \mu \mathrm{m}$ which allows to determine the refractive index better than $5 \%$.

Beside the pure geometrical data supplied by OCT, there is a lot more information achievable using PS-OCT in material testing and characterisation. As a first example, an injection-moulded polystyrene part is presented in fig. 5. Fig. 5a) shows the conventional OCT-intensity image. The cross-section of the sample looks homogenous and exhibits no distinct features, whereas a pronounced pattern appears in the simultaneously taken retardation image (fig. 5b)). 


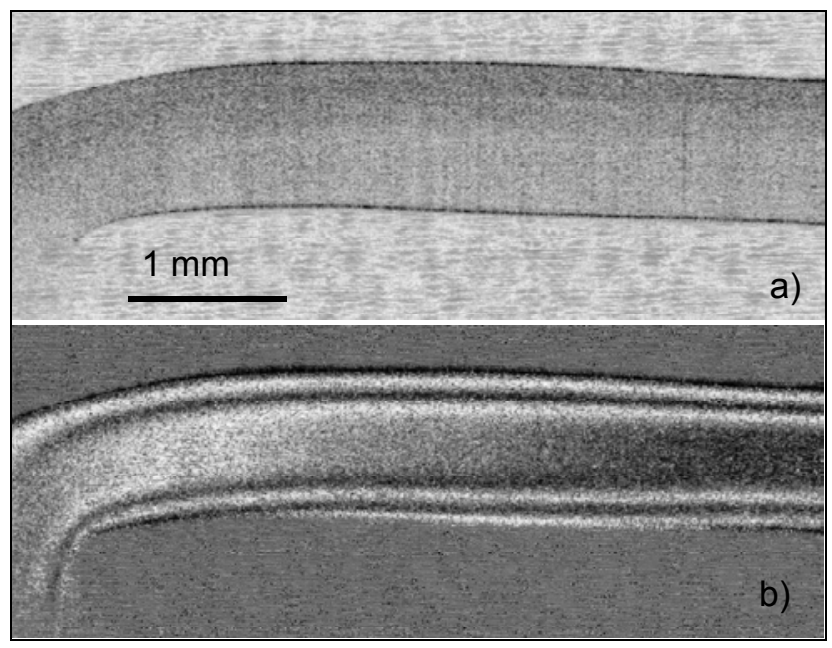

Fig. 5: PS-OCT measurements of injection moulded polystyrene part. a) OCT cross-section (intensity image), b) Polarisation-sensitive retardation image exhibiting an homogenous core and anisotropic surface regions.

In the retardation images, which are sensitive to birefringence in the material, the phase retardation between two orthogonal polarisation states of light, corresponding to ordinary and extraordinary rays of the analysing light beam, is mapped to grey levels. Thus, a transition from dark to bright indicates that the phase retardation has increased by $90^{\circ}$ over the corresponding distance within the material. In fig. 5b) the pronounced stripe pattern of the birefringence signal appears at the bottom and top surface of the sample, caused by anisotropy of the material, whereas the core region shows only slowly varying shadings, indicator for high sample homogeneity. The origin of the high anisotropy in the surface regions can be found in the increased shear forces at the interface of the mould during the injection-moulding process (polymer orientation).

Another source of birefringence in materials is stress-induced strain. The capabilities of PS-OCT to detect internal stress are exemplified by a tensile stress experiment performed on a nontransparent low density polyethylene (LDPE) sheet with a thickness of $1 \mathrm{~mm}$, as presented in fig. 6.
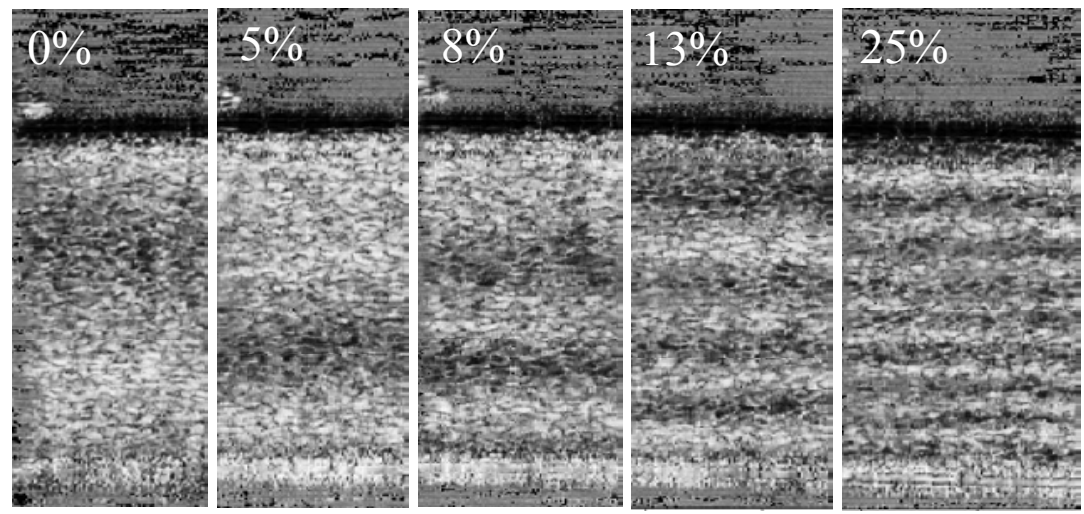

Fig. 6: Sheet of low-density polyethylene (thickness $1 \mathrm{~mm}$ ) measured with PS-OCT at different strain states (unstrained, $5 \%, 8 \%, 13 \%, 25 \%$ ). 
The sample has been subjected to tensile stress and the corresponding birefringence pattern and strain have been recorded. Each additional emerging white stripe (with increasing strain) corresponds to a further increased retardation of $180^{\circ}$. These images have been taken and the profile of the birefringence pattern has been averaged along the sample to create the profiles plotted in fig. 7 with the applied stress and resulting strain indicated.

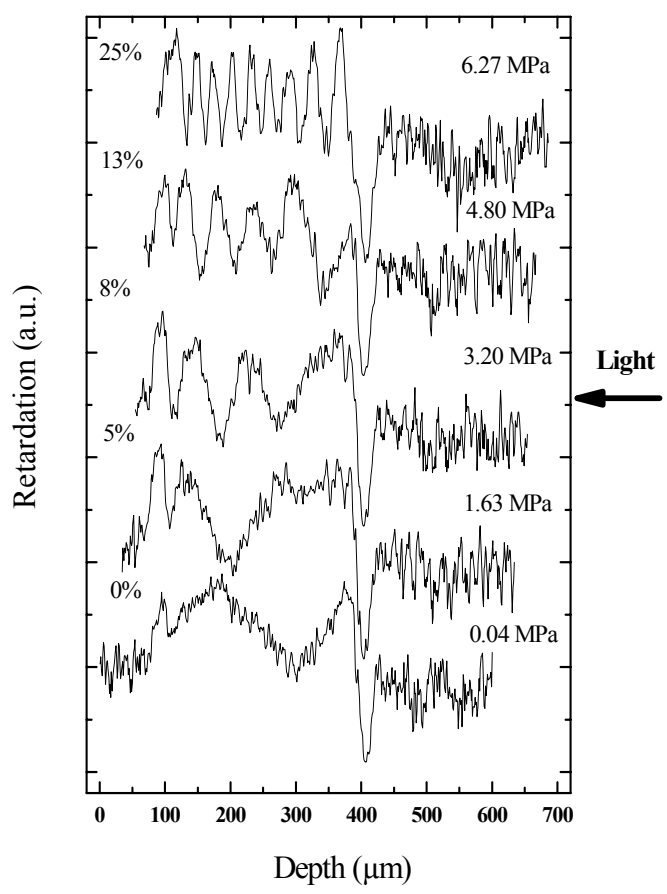

Fig. 7: Averaged retardation profiles taken from PS-OCT images of fig. 6.

Derived from experiments similar to those depicted in fig. 7, our PS-OCT setup has been calibrated for a quantitative determination of internal stress of LDPE samples in a contact-free, non-destructive and spatially resolved way: fig. 8 presents the calibration curves (stress/strain, birefringence/strain) yielding a stress optical coefficient $\mathrm{C}_{\text {opt }}=(6.3 \pm 0.3)^{*} 10^{-10} \mathrm{~Pa}^{-1}$ for the investigated LDPE.

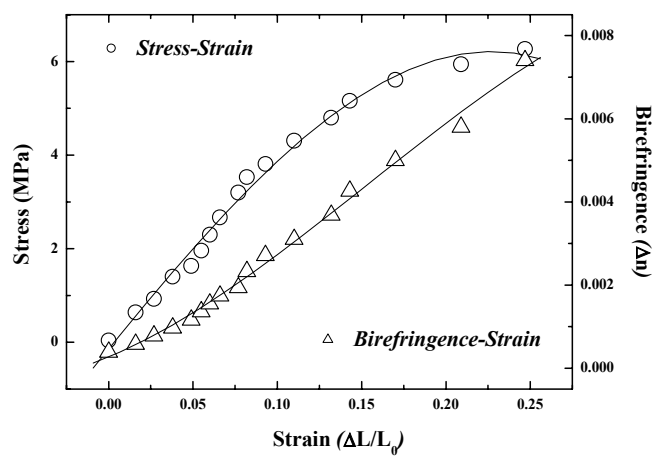

Fig. 8: Calibration curves (stress/strain, birefringence/strain) for low-density polyethylene sample of fig. 6 . 
Finally, it should be remarked that full field optical methods are up to now standard stress and strain characterisation techniques. However, there are several disadvantages to take into account. In the case of transmission photoelasticity, a scale model of the part under investigation has to be made in a suitable transparent plastic and coplanar slices of the model have to be placed into a polariscope, which renders the regions of high strain visible as an interference pattern. The fringes in these patterns can be interpreted to give information about the magnitude of the principle stresses that are present in the model. The stresses in the actual component can be derived by applying appropriate formulas on the measured data [11]. Contrary to the latter approach, PS-OCT is now capable to directly image the strain pattern in the part itself in a spatial resolved way: it is even easily possible to join consecutive $2 \mathrm{D}$ cross-section images to a full spatially resolved 3D data-cube. However, fully automated strain analysis with PS-OCT, as it is already the case for well-established photoelasticity methods, needs further developmental work.

Conclusions: OCT and PS-OCT have been used to address several problems in the area of nondestructive testing and characterization of materials down to micrometer scale. Conventional OCT has been applied to determine thickness, geometry and refractive index of layers and bulk samples. PS-OCT has been shown to be capable to map regions of material anisotropy and to determine quantitatively internal stress/strain state of materials.

Acknowledgements: Parts of this work have been carried out with financial support of the Austrian Science Fund (FWF Projects P16585-N08 and P16776-N02).

\section{References:}

[1] D. Huang, E. A. Swanson, C. P. Lin, J. S. Schuhman, W. G. Stinson, W. Chang, M. R. Hee, T. Flotte, K. Gregory, C. A. Puliafito, J. G. Fujimoto, "Optical Coherence Tomography", Science, vol. 254, pp. 1178-1181, 1991.

[2] A. F. Fercher and C. K. Hitzenberger, "Optical coherence tomography", Progr. Opt., vol. 44, pp. 215-302, 2002.

[3] B.E. Bouma and G.J. Tearney, eds., "Handbook of Optical Coherence Tomography", Marcel Dekker, New York, 2002.

[4] J. P. Dunkers, F. R. Phelan, D. P.Sanders, M. J. Everett, W. H. Green, D. L.Hunston, R. S. Parnas, "The application of optical coherence tomography to problems in polymer matrix composites", Opt. Laser Eng., vol 35, pp.135-147, 2001.

[5] M. Bashkansky, M. D. Duncan, M. Kahn, D. Lewis, J. Reintjes, "Subsurface defect detection in ceramics by high-speed high-resolution optical coherence tomography", Opt. Lett., vol 22, pp. 61- 63, 1997.

[6] J. F. de Boer, T. E. Milner, M. J. C. van Gemert, J. S. Nelson, "Two-dimensional birefringence imaging in biological tissue by polarisation-sensitive optical coherence tomography", Opt. Lett., vol. 22, pp. 934-936,1997.

[7] J.F. de Boer, T. E. Milner, "Review of polarization sensitive optical coherence tomography and Stokes vector determination", J. Biomedical Optics, vol. 7(3), pp. 359$371,2002$.

[8] C. K. Hitzenberger, E. Götzinger, M. Sticker, M. Pircher, A. F. Fercher, "Measurement and imaging of birefringence and optic axis orientation by phase resolved polarisation sensitive optical coherence tomography", Opt. Expr., vol. 9, pp. 780-790, 2001.

[9] D.Stifter, P. Burgholzer, O. Höglinger, E. Götzinger, C.K. Hitzenberger, "Polarisationsensitive optical coherence tomography for material characterisation and strain-field mapping", Appl. Phys. A 76,1-5, 2003.

[10] E.A. Swanson, D. Huang, M.R. Hee, J.G. Fujimoto, C.P. Lin, C.A. Puliafito, "High-speed optical coherence domain reflectrometry", Opt. Lett., vol. 17, pp. 151-153, 1992.

[11] A. Ajovalasit, S. Barone, G. Petrucci, "A review of automated methods for the collection and analysis of photoelastic data", J. Strain Analysis, vol. 33(2), pp. 75-91, 1998. 